\title{
A Study of the Strategy and Operation of Japanese Agriculture Cloud Computing
}

\author{
Takao Nomakuchi ${ }^{*}$, Suguru Yanata ${ }^{2}$ \\ ${ }^{1}$ Faculty of Economics, Department of Business Management, Wakayama University, Wakayama City, Japan \\ ${ }^{2}$ Faculty of Economics, Department of Economics, Wakayama University, Wakayama City, Japan \\ Email: thoma@wakayama-u.ac.jp
}

Received January 2015

\begin{abstract}
Amid declining Japanese agriculture solutions, agriculture cloud attracts much attention and expectations. However, the strategy and the operation of the agriculture cloud have not been investigated adequately. Therefore, the purpose of this study is to examine the strategy and the operation in the agriculture cloud. In this study, we performed a case study regarding agriculture cloud. Based on our consideration, the strategy and the operation of the agriculture cloud are a reinforcement of conventional competitive advantages.
\end{abstract}

\section{Keywords}

\section{Case Study, Industry Cluster, Strategic Information System}

\section{Introduction}

According to the study group for the realization of smart agriculture of the Ministry of Agriculture, Forestry and Fisheries, being innovative is important in order to continue to create an environment in which leaders can fully demonstrate their willingness and abilities. This is true even in agricultural technology and from such points of view as follows;

1) Labor-saving and light labor reduction and refinement.

2) Information technology under the severe situation where there is a shortage of farmers due to aging and very few new farmers.

3) The need to strengthen the competitiveness of agriculture, forestry and fisheries.

4) Improvement of the attractiveness of faming as an industry.

Currently, a major topic of the Trans-Pacific Economic Partnership Agreement (TPP) negotiations with the relevant countries is to advance agriculture. Outcome of negotiations as part of the agreement is quite fluid, but if liberalization is promoted, along with the idea of improving competitiveness with the use of exotic crops, then the future environment of agriculture will vary significantly. On the other hand, the robot technology and the ICT technology have advanced in other areas of agriculture, while these innovations have led to the strengthening of competitiveness, utilization of such technologies is expected to be a strong driving force in agricultural

\footnotetext{
${ }^{*}$ Corresponding author.
} 
sector for growth of the industry bringing about solutions for various agricultural issues [1].

According to a survey of Seed Planning of Japan's market research firm, the market scale of agriculture of IT is expected to be 58 billion to 60 billion yen in 2020. The growth is about nine times that of the 2013 ratio. "IT in agriculture" is defined as "by making full use of IT technology to collect information necessary for the production and sale of agricultural products, efficiently produce agricultural products and the use of technology in sales and distribution”. That incorporates high-tech areas, such as the sensing, the communication technology and the biotechnology in agriculture. Above all, it will be agriculture with cloud services provided by municipalities and major IT vendors that should produce significant progress. The growth should increase to about 28 times that of the 2013 ratio and account for $75 \%$ of the agricultural IT market [2].

Therefore, the challenge is to achieve the effective use of the agricultural cloud. It requires the capture of the future needs created in agricultural production sites and the distribution process in order to develop the strategy and the operation of the services and the technologies needed. However, the strategy and the operation are not revealed in the agriculture cloud at the moment. Therefore, the purpose of this study is to examine the strategy and the operation of the agriculture cloud. However, the events of an agricultural business cycle does not occur only once in a year. It will take an extended period to verify the usefulness of such systems in farming for the agriculture cloud development. Therefore, we reviewed the current state of the related works of Japanese agriculture cloud, and considered the strategy and the operation from an useful case that utilize the cloud in agriculture at the present time.

\section{Related Work}

In this chapter, we reviewed the related works on the use of IT in agriculture, and then considered the related works on cloud computing in agriculture. We also considered the works related to the cloud strategy.

\subsection{Agriculture IT}

First, the related work on the utilization of IT in agriculture is considered here. The reference [3] considers the IT utilization in Japan of agriculture, based on the demonstration experiments they conducted. Most of the information technology (IT) systems currently used by Japanese farmers are used for duties such as filing tax records and traceability of records to meet the requirements of non-farmers in the government and distribution industry. They focused on introducing the latest technologies such as sensors, wireless networks, and cloud computing in order to radically revise approaches in agriculture and conduct business feasibility studies to establish a hypothetical model so that Cloud services could make genuine contributions to agriculture. Demonstration tests have been conducted with the cooperation of two Japanese farming corporations. This hypothetical model has much in common with on-site operations such as medical/nursing care and maintenance, besides agriculture. Therefore, they are working to develop it across a broader range of fields. They used Web applications and mobile phone applications were used to build prototypes for the four functions listed below. Verification the experiments were performed with the cooperation of farming corporations at two locations in Japan (a vegetable farm in Miyazaki Prefecture, and a rice paddy/dry field farm in Shiga Prefecture). These experiments were executed to verify their effectiveness from technical and business points of view.

1) Sales/planting (production) planning

Sales to customers and planting (production) planning for cultivated land can be performed together.

2) Operational planning/results management

Progress management and operational checks can be performed on the basis of preplanned on-site work and automatic collection of results.

3) Patrolling support

Reports and instructions can be easily and reliably issued by sharing on-site photographs and comments among all administrators and workers.

4) Cultivated land data management

Management of all sorts of data relating to cultivated land, including location, land rights, area, soil, and land characteristics can be integrated.

These four functions are supported by Data storage and Data Analysis, which are based on technologies such as sensors (weather, soil, global positioning system [GPS]), networks (wireless local area network, third generation [3G]), and knowledge management. The above functions should eventually make it possible to add func- 
tions to address various different requests from on-site workers.

In this related work, by making the field of agriculture similar to industrial plants, profitability acquisition by efficiency and quality improvement by the use of IT has been proposed.

\subsection{Cloud Computing for Agriculture}

Second, the related work of cloud computing utilization in agriculture is reviewed. According to the reference [4], by providing a mechanism for an integrated management of plant factories with the cloud services, information for the cultivation environment control is stored. Agricultural crop failure factors can become apparent using time series analysis of this information. It is possible to perform the service of plant factories operational support for farms. In addition, it is to collect the various needs of the demand side, such as retailers, consumers, processors and distributors in a timely manner, by telling them information concerning the farmer who is a producer. As a result, farmers can increase or decrease the production of agricultural crops. The needs of the demand side can be acquired enabling the farmer to reproduce "sell crops". In other words, building the IT foundation where a broad range of data can be shared between producers and consumers can promote social infrastructures for agriculture. The result generates a smart future industry for "food". Therefore, plant factory production support cloud services are proposed. Currently, in order to achieve a stable supply of agricultural products, and to properly control the growth environment in accordance with agricultural needs, installation of plant factories to achieve stable production is increasing. As a result, monitor the growing capacity utilization status and growth status remotely enables optimization and various data analysis of environmental controls. Required controls are such things as energy control, and services to assist in the improvement of productivity. Plant factory production support cloud services are enlarging the environmental data and services to integrate and manage the cultivation equipment control data to the cloud environment for the plant factory. By providing control information in real time for producers and equipment managers, it also provides a service that enables control of the growing environment and cultivation equipment. This service, by controlling the growing environment in plant factories, helps improve the quality and productivity of the plant to produce. It is also a service of the full-scale plant for the factory to provide a variety of analytical information. First, from the sensor equipment and control devices, the amount of solar radiation, temperature, humidity, nutrients, $\mathrm{pH}$, EC (Electrical Conductivity) growing environment data, such as air conditioning and heating facilities and nutrient solution pump, operation of cultivation equipment, such as curtains status data, are collected in the plant factory. Afterward, through the data collection and storage and distribution infrastructure in the data center, such data is displayed on a monitor for the administrator on a real-time basis. It is also possible to remotely configure the control settings for cultivation equipment and growing environment data collection sensor devices. In addition, all data is stored in a database and analyzed by using BI (Business Intelligence) tools. As a result decision support from growing environment optimization and management point of view is realized. As for solutions that support the next generation agriculture, they have proposed a service to collect environmental data and control data of the plant factory, to visualize in cloud computing, and to help the farmers. According to this related work in agricultural areas, where the IT utilization has yet to be used, business reform and efficiency through the use of cloud computing has been proposed.

\subsection{Things Discovered from Related Works}

The related works of the IT strategies in the industries are overviewed. In the case of customers and travel agency concerning airline reservation, Wiseman proposed a strategic information system (SIS). SIS is an IT concept using strategic tool to create customers by creating customer value [5]. So far, since IT has not been applied in agriculture, we thought that it is necessary at this time to start applying it by using the SIS concept. Moreover, cloud computing is considered to be a service that provides computing resources and application software by using the Internet technology. According to Michael Porter, Internet technology becomes a powerful source for a competitive advantage only by integrating a business strategy. The Internet itself is also a neutral presence in terms of a source of competitive advantage. Without the Internet, companies cannot continue to participate in the game; however, not only Internet companies can obtain competitive advantages. Strong competitive advantages come from the achievements of conventional strengths. They are unique products and content, and special production activities, superior product knowledge, and a powerful anti-personnel service along with customer relationships. Strategies, such as the integration of the Internet and conventional competitive advan- 
tages and competitive approach, have been successful in many industries [6]. In addition, when viewed from the perspective of the industrial cluster theory, it is unlikely that farmers can build competitive advantages by themselves. Industrial cluster composed by suppliers, distributors and food manufacturers are important for farmers. In recent years, concentrated communication and flexibility of networks in industrial clusters have produced innovation [7]. According to related works, the concept and points to regarding the strategies of agricultural cloud is summarized in Table $\mathbf{1}$ below. From these points, and by considering these cases, the required strategies and operations are considered in agriculture cloud.

\section{Case Study}

\subsection{Calbee and Calbeepotato Overview}

In this chapter, the subject of the case study is Calbee Corporation (referred to as Calbee hereinafter) and Calbeepotato Inc. (referred to as Calbeepotato hereinafter). Calbeepotato is a procurement specialist subsidiary of Calbee. Overviews of Calbee and Calbeepotato are as follows;

Calbee was established in April, 1949. The business category of this company is grocery. This company's business contents include the manufacturing and sales of cornflakes. "Utilizing the idea of cherishing nature's bounty, we created delicious and fun foods to contribute to the healthy living of people.” This is the Calbee Group's corporate philosophy. As new products were developed, they launched Kappaebisen, potato chips, Furugura, Jagariko, and Jagabee every 10 years. Calbee has grown as a brand that Japanese consumers are familiar with. Calbee has developed potatoes as raw materials as the foundation for the development of these products. Procurement and manufacturing have been built by integrating a value chain leading up to the sales over-thecounter. In addition, Calbee not only takes care of new needs and the value of customers, but also tries to reflect management issues and valuable opinions as well as requests of our customers as to their own business activities. Since its foundation, Calbee has developed unique products and built a thorough quality control system, such as placing the production date on each product label [8].

Calbeepotato is a subsidiary of Calbee. This company broke off from the Raw Material Department of Calbee Calbee and became independent in October, 1980. Calbeepotato purchases, stores, transports, and sells potatoes and other agricultural products. In addition, development, production, and sales of other potato products and agricultural processed products are included. As for the business policies, as a leading company that opened up Japan's potato industry, this company has developed a new food culture from potatoes. By introducing the most advanced technology in the field of "agriculture" which is based on nature, they have focused on the development of strong and better varieties with advantages over various potato-related diseases and disorders. Furthermore, by advancing the development of processing products according to consumers' demand, they increased the availability of potatoes. All potatoes are procured through contracted farmers across the nation. Procured potatoes are divided and separated according to each farmer, brand, and each planting field. By doing so, they have carried out strict quality control at each stage of the distribution to customers. The consistent system of storage from the production to logistics is unique in the world. Therefore, for consumers, farmers and processing manufacturers, this company provides a new food culture based on potatoes. By using natural potatoes, Calbeepotato has contributed to health promotion and people's lives [9]. The agricultural cloud case of the Calbee group is discussed. In this paper, Calbee and Calbeepotato are referred to as the Calbee group.

\subsection{Agriculture Cloud Case of Calbee Group}

"Potato chips" and "Jagariko" of Calbee are long-selling snack products. However, the problem is a lack of potatoes as a raw material. Due to import restrictions on raw potatoes in Japan, for the Calbee, it is important to procure high quality of potatoes in the country. According to Calbee, cultivation and efforts by agricultural

Table 1. The strategy and the operation of cloud.

\begin{tabular}{cc}
\hline Concepts & Points to Consider \\
\hline Strategic Information System (SIS) & $\begin{array}{c}\text { The create customer value, and to create customers. } \\
\text { Integration of Cloud and conventional competitive } \\
\text { advantages and competitive approaches }\end{array}$ \\
Industrial cluster & Innovation created by concentrated communication and flexibility of network \\
\hline
\end{tabular}


technology that surfaced have changed the quality of fertilizers and soil used for growing potatoes, but agricultural support using IT has almost never been provided in Japan. Since 2006, Calbee has used the agricultural cloud service in the production process management of potatoes as a raw material for their core products, such as "potato chips" and "Jagariko". The installation of facilities called "weather station" is an example. This is a measuring system for the cultivation conditions, such as the amount of wind, daylight hours, temperature, humidity, water content in the soil, and temperature on a real-time basis. The data is stored in the agriculture cloud. By analyzing the data from the agriculture cloud, Calbeepotato aims to optimize cultivation methods for contracted potato farmers. By having many contract farmers use this system, the cost of agriculture cloud is maintained low. Other ways of using the agricultural cloud service is to divide the potato-planting processes into 10 steps, while each of these steps is recorded in cloud so that they can be checked. By using the agricultural cloud data, knowledge is produced using planting innovation, while farmers are able to share such knowledge on the cloud. Since 2006, the Calbee group has made these efforts using the Project of Quality Assurance on the Total Amount of Potatoes. For example, time to plant seed tubers, spraying time and use of pesticides, weather and water conditions are recorded. As a result, the quality and yield of the harvested potatoes are recorded, and provides leverage in order to innovate cultivation methods. The purpose of this effort is to utilize agricultural cloud in agriculture businesses, so that the hypotheses can be verified. For example, such hypotheses include "increasing the width of the field ridges" and "to partial use new pesticide". Cultivation method data to be recorded is then collected from farmers by individuals in charge of Calbeepotato. Other types of cultivation data, such as weather, temperature, humidity, sun exposure, and the underground temperature is collected by the weather stations. This agriculture cloud utilization is assumed to be intended to support and strengthen the long-standing business of Calbeepotato personnel. The Calbee group has established potato cultivation theories through their past experience. Each person in charge has given advice and counsel to contracted farmers. Based on these efforts, contracted farmers have accumulated overall know-how by utilizing agricultural cloud. Previously, because individual were in charge of carrying out operations, knowledge sharing was impossible. Agriculture cloud with the focus on these weather stations is intended to further enhance the knowledge in potato cultivation operations. This is the strength of Calbee. This strength has led to quality improvement and differentiation of products. In addition, Calbee holds study sessions for the local farmers each New Year. There farmers present the current knowledge on potato cultivation, and that supports networking among farmers by communication. One contracted farmer made the following comment, "Farmers do not know what other farmers are doing. It's quite a rare case to talk with other farmers about potato cultivation methods. But the individuals in charge from Calbee take a neutral position, while showing some effective cultivation methods. So I think this lecture is quite valuable." Calbeepotato appoints about 30 individuals in charge of this group across the country. Based on data in the database, they visit and advise farmers once a week. In addition, in agreement with the farmers, Calbee changes the purchase price by the quality of the potatoes. By conducting sampling inspections to check potatoes suffering from a disease, they purchase healthy potatoes at high prices. Initially there were some conflicts; however, hard-working farmers are well paid, while all of capable farmers were improved. Factors that have enabled these activities include the presence of trustful relationship between the Calbee and farmers. Some 30 years ago, Calbee supported farmers in the establishment of a potato processing production union. Then 30 years later, they work together, and have improved the quality of potatoes [10]. In 2013, Calbee has averted poor harvest of potatoes by analyzing the automatically collected data via sensors as well as manually. In June 2013, Calbeepotato sent warning messages to about 1100 mobile phones and smartphones of farmers in order to let them know the need to take countermeasures for potential potato diseases which would appear to begin happening within two weeks. This was done based on the data that has accumulated in the cloud and calculated by using the mathematical model for calculation provided by the specialized institutions, the "Hokkaido Pest Control Institute". According to the contracted farmers, the pestilence prediction information is more accurate and reliable. It also leads to the prevention of human errors such as overlooked accidents in the growth process and inappropriate methods of farming. One of the examples is the optimum depth to inculcate seed potatoes in the soil. From the cloud of data, the relationship with the seed tuber planting depth and potato harvest quality is analyzed. From the analysis, the following points have been found out. When the planting depth of the seed tuber is shallower than $15 \mathrm{~cm}$, potatoes become green and uneatable. When it is too deep, the seed tubers spoil. As a result, if it is deeper than $17 \mathrm{~cm}$, the risk of spoilage increases. A difference of only a few centimeters becomes the turning point to cultivate qualify potatoes as a raw material. In three years, the amount of green potatoes was cut in half. 
Additionally, the following was also found. After two months from planting of seed tubers, if the length of the stem has not reached $20 \mathrm{~cm}$, potatoes do not grow properly. The length of the stems of potatoes usually extends up to $70 \mathrm{~cm}$ on average. From this discovery, at the stage where the length of the stem is not less than the half, contract farmers can now discover the growth abnormalities of potatoes. For poor growth is found at an early stage, contract farmers can now improve the poor growth of potatoes by, for example, by giving fertilizer. From the results of such efforts, in 2011-2013, the potato harvest amount of Hokkaido increased steadily [11].

\subsection{Summarization of the Case Study}

According to this case study, the strategy and the operation of the agriculture cloud by the Calbee group are shown in Table 2 below.

In the Calbee group, using agriculture cloud, the existing operation of the contract farmers and the individuals in charge has been advanced. A high-quality potato produces customer value. The agriculture cloud has integrated into the operations of farmers, Calbeepotato and Calbee. It has further strengthened the existing competitive advantage of the Calbee group.

In addition, the concentrated communication and network flexibility of the potato farmers and innovation in cultivation methods have been created. In addition, in order to create farmers innovation by agriculture cloud, it is necessary for Calbeepotato personnel to support such features. By their presence, agricultural cloud of the Calbee group worked strategically where the operation is carried out.

\section{Conclusion}

The purpose of this study was to consider the agriculture cloud strategy and operation, along with conducting the case study. Based on our consideration, the strategy of the agriculture cloud is not a transformation a agricultural land to a factory through the use of IT for efficiency. We can draw conclusions as follows;

- Agricultural cloud produces the value for consumers and food manufacturers, and creates demand.

- Agricultural cloud reinforces conventional competitive advantages in the cloud.

- Agricultural cloud creates innovation by concentrated communication and flexibility of the network.

- The agricultural cloud operations are as follows.

- Based on the data, agricultural cloud performs pestilence forecasts and growth forecast.

- In the cloud data, agricultural cloud creates a flexible network and concentrated communication within the agriculture cluster.

- Agricultural cloud sets up human support.

The future issues are as follows; Conducting questionnaire surveys targeting farmers, we need to consider the effect and improvements of the agriculture cloud. While developing the agriculture cloud introduction methodology, we have to conduct training of the support staff members.

Table 2. The strategy and the operation of agriculture cloud.

\begin{tabular}{ccc}
\hline Concepts & Points to Consider & Agriculture cloud of the Calbee group \\
\hline $\begin{array}{c}\text { Strategic Information } \\
\text { System (SIS) }\end{array}$ & $\begin{array}{c}\text { The creation of customer } \\
\text { value, and to creation of } \\
\text { customers. }\end{array}$ & $\begin{array}{c}\text { By ensuring the harvest high quality potatoes and } \\
\text { value for the consumer is created. }\end{array}$ \\
$\begin{array}{c}\text { The competitive } \\
\text { advantage via the } \\
\text { Cloud }\end{array}$ & $\begin{array}{c}\text { Integration of the Cloud } \\
\text { and conventional } \\
\text { competitive advantage and } \\
\text { competitive approach }\end{array}$ & $\begin{array}{c}\text { Advice given to farmers by Calbeepotato personnel } \\
\text { becomes scientific based on the data from cloud. } \\
\text { Furthermore, to perform new operations such } \\
\text { pestilence forecasts and growth predictions. }\end{array}$ \\
$\begin{array}{c}\text { Industrial cluster } \\
\text { Innovation created by } \\
\text { concentrated }\end{array}$ & $\begin{array}{c}\text { Using the agriculture cloud, it can be the hub of a } \\
\text { communication and } \\
\text { flexibility of networks }\end{array}$ & $\begin{array}{c}\text { farmers can connect with each other. New cultivation } \\
\text { methods are created by this close communication. }\end{array}$ \\
\hline
\end{tabular}




\section{Acknowledgements}

The information sources for this case study are public and provided by Calbeepotato. Taking advantage of this opportunity, we would like to express our appreciation.

\section{References}

[1] Ministry of Agriculture, Forestry and Fisheries (2014) For the Interim Report of the "Study Group for the Realization of the Smart Agriculture” Study Results. http://www.maff.go.jp/j/kanbo/kihyo03/gityo/g_smart_nougyo/

[2] Seed Planning (2014) Market Forecast of the Agriculture of IT. Seed Planning Press Release, 2014/01/09. https://www.seedplanning.co.jp/press/2014/2014010901.html

[3] Hori, M., Kawashima, E. and Yamazaki, T. (2010) Application of Cloud Computing to Agriculture and Prospects in Other Field. FUJITSU Scientific \& Technical Journal, 46, 446-454. http://www.fujitsu.com/downloads/MAG/vol46-4/paper15.pdf

[4] Shimizu, S., Sugihara, N., Wakizaka, N., Oe, K. and Katsuta, M. (2014) Plant Factory Production Support Cloud Services for the Next Generation of Agricultural Business. Hitachi Hyoron, 96, 652-653. http://www.hitachihyoron.com/2014/10/

[5] Wiseman, C. (1988) Strategic Information Systems. McGraw-Hill Professional, Water Street.

[6] Porter, M.E. (2001) Strategy and the Internet. Harvard Business Review, 79, 62-78.

[7] Saxenian, A. (1996) Regional Advantage: Culture and Competition in Silicon Valley and Route 128. Harvard University Press, Boston.

[8] Calbee HomePage. https://www.calbee.co.jp/english/

[9] Calbeepotato HomePage. http://www.calbee-potato.co.jp/

[10] Potatoes of the Underlying Strength—Core Business Innovation Strategy of the Calbee. Nikkei Computer 2006, December, 25th, 69-77.

[11] Calbee Potato Data Scientist. Nikkei Information Strategy, December 2013, 26-29. 